International Mathematical Forum, Vol. 8, 2013, no. 4, 181 - 190

\title{
On Fuzzy Subgroups of Finite Abelian Groups
}

\author{
Bashir Humera and Zahid Raza
}

\author{
Department of Mathematics \\ National University of Computer \& Emerging Sciences \\ Lahore Campus, Pakistan. \\ humerajam@hotmail.com, zahid.raza@nu.edu.pk
}

\begin{abstract}
In this paper,we determine the number of fuzzy subgroups of finite abelian group $G=Z_{p_{1}} \times Z_{p_{2}} \times \cdots \times Z_{p_{n}}$, where $p_{1}, p_{2}, \ldots, p_{n}$ are all distinct primes. We have used the equivalence relation on the fuzzy subgroups of any group $G$ defined by Sulaiman and Abdul Ghafur [3]in order to determine all fuzzy subgroups of the above mentioned group. We also determined the number of fuzzy subgroups of abelian group $G=Z_{p^{n}} \times Z_{q}$ under the same equivalence relation.
\end{abstract}

Mathematics Subject Classification: 05-xx,15-xx, 15B36, 15A36

Keywords: partial ordered set, equivalence ,lattice, chain, fuzzy subgroup

\section{Introduction}

In 1965, Zadeh first introduced fuzzy set, while Mordeson et.al called him a pioneer of work on fuzzy subsets. After that paper, several aspects of fuzzy subsets were studied.In 1971, Rosenfeld [11] introduced fuzzy sets in the realm of group theory and formulated the concepts of fuzzy subgroups of a group. An increasing number of properties from classical group theory have been generalized.

One of the most important problem of fuzzy theory is to classify the fuzzy subgroups of a finite group[4]. Several problems have treated the particular case finite abelian group. Laszlo studied the construction of fuzzy subgroups of groups of the orders one to six. Zhang and Zhou[14] have determined the number of fuzzy subgroups of cyclic groups of the order $p^{n}$ where $\mathrm{p}$ is a prime number. Murali and Makamba [8], considering a similar problem, found the number of fuzzy subgroups of groups of the order $p^{n} q^{m}$ where $\mathrm{p}$ and $\mathrm{q}$ are distinct primes. In [13], Tarnauceanu and Bentea established the recurrence 
relation verified by the number of fuzzy subgroups of finite cyclic groups. Their result is improving Murali's work in [10, 9].

Raden Sulaiman and Abd Ghafur Ahmed [3], worked on the particular case of finite abelain cyclic groups $G=Z_{p} \times Z_{q} \times Z_{r} \times Z_{s}$, namely with $p, q, r, s$ are distinct prime numbers. However, their approach is different from Tarnauceanu in [13]. Using their approach, we have generalized the case of fuzzy subgroups of finite abelian groups $G=Z_{p_{1}} \times Z_{p_{2}} \times \cdots \times Z_{p_{n}}$ where $p_{1}, p_{2}, \ldots, p_{n}$ are all distinct primes.

\section{Preliminaries}

In this section we summarize the preliminary definition and result that are required later in this paper. In this section, a group $G$ is assumed to be a finite group.

Definition 2.1 Let $X$ be a nonempty set. A fuzzy subset of $X$ is a function $\mu$ from $X$ into $[0,1]$.

Definition 2.2 A fuzzy subset of $G$ is called a fuzzy subgroup of $G$ if

$$
\begin{gathered}
\mu(x y) \geq \min \{\mu(x), \mu(y)\}, \quad \forall x, y \in G \\
\mu\left(x^{-1}\right) \geq \mu(x), \quad \forall x \in G .
\end{gathered}
$$

Theorem 2.3 [4]. A fuzzy subset $\mu$ of $G$ is a fuzzy subgroup of $G$ if and only if there is a chain of subgroups of $G$,

$$
P_{1}(\mu) \leq P_{2}(\mu) \leq \ldots \leq P_{n}(\mu)=G
$$

such that $\mu$ can be written as

$$
\mu(x)= \begin{cases}\theta_{1}, & x \in P_{1}(\mu) \\ \theta_{2}, & x \in P_{2}(\mu) \backslash P_{1}(\mu) \\ \vdots & \\ \theta_{n}, & x \in P_{n}(\mu) \backslash P_{n-1}(\mu)\end{cases}
$$

Example 2.4 Consider the group $G=Z_{48}$. Define functions $\mu, \gamma, \alpha, \beta$ as follows :

$$
\begin{gathered}
\mu(x)= \begin{cases}1, & x \in\{0,4,8,12,14,16,20,22\} \\
\frac{2}{5}, & x \in\{1,2,3,5,6,7,9,10,11,13,15,17,19,21\}\end{cases} \\
\gamma(x)= \begin{cases}1, & x \in\{0,6,12,18,24,30,36,42\} \\
\frac{1}{2}, & x \in\{2,4,8,10,14,16,20,22,26,28,32,34,38,40,44,46\} \\
\frac{1}{4}, & x \in\{1,3,5,7,9,11,13,15,17,19,21, \ldots, 47\}\end{cases}
\end{gathered}
$$




$$
\begin{aligned}
& \alpha(x)= \begin{cases}\frac{1}{2}, & x \in\{0,4,8,12,16,20,24,28,32,36,40,44\} \\
\frac{2}{3}, & x \in\{2,6,10,14,18,22,26,30,34,38,42,46\} \\
0, & x \in\{1,3,5,7,9,11,13,15,17,19,21,23, \ldots, 47\}\end{cases} \\
& \beta(x)=\left\{\begin{array}{cl}
1, & x \in\{0,4,8,12,16,20,24,28,32,36,40,44\} \\
\frac{1}{2}, & x \in\{2,6,10,14,18,22,26,30,34,38,42,46\} \\
\frac{1}{4}, & x \in\{1,3,5,7,9,11,13,15,17,19,21,23, \ldots, 47\}
\end{array}\right.
\end{aligned}
$$

Note that $P_{1}(\mu)=\{0,4,8,12,14,16,20,22\}$ is not a subgroup of $Z_{48}$. According to Theorem $2.3, \mu$ is not a fuzzy subgroup of $Z_{48}$, where as $\alpha, \beta$ and $\gamma$ are fuzzy subgroups of $Z_{48}$.

\section{$3 \quad$ Fuzzy Subgroups of $G=Z_{p_{1}} \times Z_{p_{2}} \times \cdots \times Z_{p_{n}}$}

Since without any equivalence relation on fuzzy subgroups of group $G$, the number of fuzzy subgroups is infinite, even for the trivial group $\{e\}$. So $[3,4]$ defined the equivalence relation on the set of all fuzzy subgroups of $G$.This Definition differs from that of Murali and Makamba [4,5], but it is equivalent to the Definitions of Dixit [11],Zhang [8] and Tarnauceanu [7]. We prefer to use this Definition given below:

Definition 3.1 [4]. Let $\mu, \gamma$ be fuzzy subgroups of $G$ of the form

$$
\mu(x)=\left\{\begin{array}{ll}
\theta_{1}, & x \in P_{1} \\
\theta_{2}, & x \in P_{2} \backslash P_{1}(\mu) \\
\vdots & \\
\theta_{m}, & x \in P_{m} \backslash P_{m-1}(\mu)
\end{array}, \gamma(x)= \begin{cases}\lambda_{1}, & x \in M_{1} \\
\lambda_{2}, & x \in M_{2} \backslash M_{1}(\gamma) \\
\vdots & \\
\lambda_{n}, & x \in M_{n} \backslash M_{n-1}(\gamma)\end{cases}\right.
$$

with

$\theta_{i}, \lambda_{j} \in[0,1], \theta_{k}>\theta_{l}, \lambda_{k}>\lambda_{l} \quad$ for $\quad k<l$ and $\bigcup_{1 \leq i \leq m} P_{i}=G, \bigcup_{1 \leq j \leq n} M_{j}=G$.

Then we define that $\mu$ and $\gamma$ are equivalent if $m=n$ and $A_{i}=B_{i}, \forall i \in$ $\{1,2,3, \ldots, n\}$.

It is easy to check that this relation is indeed an equivalence relation. Two fuzzy subgroups of $G$ are said to be different if they are not equivalent.

Example 3.2 Let $\alpha, \beta, \gamma$ be fuzzy subgroups as in example 2.4.

Since $P_{1}(\gamma) \neq P_{1}(\beta), \forall i \in 1,2,3$. Thus $\alpha$ is equivalent to $\beta$ whereas $\gamma$ is not equivalent to $\beta$.

Lemma 3.3 [3] The number of fuzzy subgroups is equal to the number of chain on the lattice subgroups of $G$. 
Theorem 3.4 [3] Let $H$ be a subgroup of $G$, and let the set of all subgroups of $G$ which contain $H$ (but are not equal to $H$ ) be $H_{1}, H_{2}, H_{3}, \ldots, H_{n}=G$. Then

$$
O\left(F_{p_{1}=H}\right)=\sum_{i=1}^{n} O\left(F_{p_{1}=H_{i}}\right)
$$

Theorem 3.5 [3] Let e be identity element of a group G. Then

$$
O\left(F_{G}\right)=2 . O\left(F_{p_{1}=\{e\}}\right)
$$

Theorem 3.6 [3] Let $G=Z_{p} \times Z_{q} \times Z_{r} \times Z_{s} \times Z_{t}$, where $p, q, r, s$ and $t$ are distinct primes. Then the number of fuzzy subgroups of $G$ are 1080.

Proof: Since the oder of the group $G=Z_{p} \times Z_{q} \times Z_{r} \times Z_{s} \times Z_{t}$ is pqrst. Hence every nontrivial subgroups of $G$ must be of order

$$
\begin{aligned}
& p, q, r, s, t, p q, p r, p s, p t, r, q s, q t, r s, r t, p q r, p q, p q t, p r s, \\
& \text { prt,pst, qst, rst, qrt, pqrs, pqrt, pqst, prst, qrst. }
\end{aligned}
$$

Hence every subgroup of $G$ can be obtained from $G=Z_{p} \times Z_{q} \times Z_{r} \times Z_{s} \times Z_{t}$ by changing $k$ pieces of $Z_{p}, Z_{q}, Z_{r}, Z_{s}$ and $Z_{t}$ to be 0 , where $0 \leq k \leq 5$. For $k=0$, the subgroup is $G$ and for $k=n$ that subgroup is $\{e\}=\{0\} \times\{0\} \times$ $\{0\} \times\{0\} \times\{0\}$. Thus we have 30 nontrivial subgroups of $G$, namely

$H_{1}=Z_{p} \times Z_{q} \times Z_{r} \times Z_{s} \times\{0\}, H_{2}=Z_{p} \times Z_{q} \times Z_{r} \times\{0\} \times Z_{t}$ $H_{3}=Z_{p} \times Z_{q} \times\{0\} \times Z_{s} \times Z_{t}, H_{4}=Z_{p} \times\{0\} \times Z_{r} \times Z_{s} \times Z_{t}$ $H_{5}=\{0\} \times Z_{q} \times Z_{r} \times Z_{s} \times Z_{t}$

$H_{6}=Z_{p} \times Z_{q} \times Z_{r} \times\{0\} \times\{0\}, H_{7}=Z_{p} \times Z_{q} \times\{0\} \times Z_{s} \times\{0\}$ $H_{8}=Z_{p} \times\{0\} \times Z_{r} \times Z_{s} \times\{0\}, H_{9}=\{0\} \times Z_{q} \times Z_{r} \times Z_{s} \times\{0\}$ $H_{10}=Z_{p} \times\{0\} \times Z_{r} \times\{0\} \times Z_{t}, H_{11}=Z_{p} \times\{0\} \times\{0\} \times Z_{s} \times Z_{t}$ $H_{12}=\{0\} \times Z_{q} \times Z_{r} \times Z_{s} \times\{0\}, H_{13}=\{0\} \times Z_{q} \times Z_{r} \times\{0\} \times Z_{t}$ $H_{14}=\{0\} \times Z_{q} \times\{0\} \times Z_{s} \times Z_{t}, H_{15}=\{0\} \times\{0\} \times Z_{r} \times Z_{s} \times Z_{t}$ $H_{16}=Z_{p} \times Z_{q} \times\{0\} \times\{0\} \times\{0\}, H_{17}=Z_{p} \times\{0\} \times\{0\} \times Z_{s} \times\{0\}$ $H_{18}=Z_{p} \times\{0\} \times Z_{r} \times\{0\} \times\{0\}, H_{19}=Z_{p} \times\{0\} \times\{0\} \times\{0\} \times Z_{t}$ $H_{20}=\{0\} \times Z_{q} \times\{0\} \times Z_{s} \times\{0\}, H_{21}=\{0\} \times Z_{q} \times Z_{r} \times\{0\} \times\{0\}$ $H_{22}=\{0\} \times Z_{q} \times\{0\} \times\{0\} \times Z_{t}, H_{23}=\{0\} \times\{0\} \times Z_{r} \times Z_{s} \times Z_{t}$ $H_{24}=\{0\} \times\{0\} \times\{0\} \times Z_{s} \times Z_{t}, H_{25}=\{0\} \times\{0\} \times Z_{r} \times Z_{s} \times\{0\}$ $H_{26}=Z_{p} \times\{0\} \times\{0\} \times\{0\} \times\{0\}, H_{27}=\{0\} \times Z_{q} \times\{0\} \times\{0\} \times\{0\}$ $H_{28}=\{0\} \times\{0\} \times Z_{r} \times\{0\} \times\{0\}, H_{29}=\{0\} \times\{0\} \times\{0\} \times Z_{s} \times\{0\}$ $H_{30}=\{0\} \times\{0\} \times\{0\} \times\{0\} \times Z_{t}$

According to [3], we have $O\left(F_{P_{1}=G}\right)=1$ and

$$
O\left(F_{P_{1}=H_{1}}\right)=O\left(F_{P_{1}=H_{2}}\right)=O\left(F_{P_{1}=H_{3}}\right)=O\left(F_{P_{1}=H_{4}}\right)=O\left(F_{P_{1}=H_{5}}\right)=1
$$




$$
\begin{gathered}
O\left(F_{P_{1}=H_{6}}\right)=O\left(F_{P_{1}=H_{7}}\right)=\cdots=O\left(F_{P_{1}=H_{15}}\right)=3 \\
O\left(F_{P_{1}=H_{16}}\right)=O\left(F_{P_{1}=H_{17}}\right)=\cdots=O\left(F_{P_{1}=H_{25}}\right)=13
\end{gathered}
$$

and

$$
O\left(F_{P_{1}=H_{26}}\right)=O\left(F_{P_{1}=H_{27}}\right)=\cdots=O\left(F_{P_{1}=H_{30}}\right)=75
$$

Then by [3], we obtain

$$
O\left(F_{P_{1}=\{e\}}\right)=5 \times 75+5 \times 1+10 \times 3+10 \times 13=540
$$

and therefore by 3.5,

$$
O\left(F_{G}\right)=2 \times 540=1080
$$

Theorem 3.7 Let $G=Z_{p_{1}} \times Z_{p_{2}} \times \cdots \times Z_{p_{n}}$ where $p_{1}, p_{2}, \ldots, p_{n}$ are $n$ distinct primes. Then the number of subgroups of $G$ present in any layer of subgroup lattice of $G$ are ${ }^{n} C_{r}, 1 \leq r \leq n$, where $n$ is number of distinct primes in a group $G$ and $r$ denotes the $r^{\text {th }}$ layer on the subgroup lattice of the group.

Proof: Since order of $G$ is $p_{1}, p_{2}, \ldots, p_{n}$, where all these $p_{i}^{\prime} s$ are $n$ distinct primes. Then the non-trivial subgroups of $G$ must be of order

$$
\begin{gathered}
p_{1}, p_{2}, \ldots, p_{n}, \\
p_{1} p_{2}, p_{1} p_{3}, \ldots, p_{n-1} p_{n}, \\
p_{1} p_{2} p_{3}, p_{1} p_{3} p_{4}, \ldots, p_{n-2} p_{n-1} p_{n}, \\
\vdots \\
p_{1} p_{2} \ldots p_{n-1}, \ldots, p_{2}, p_{3}, \ldots, p_{n},
\end{gathered}
$$

Therefore, the non-trivial subgroups of $G$ that we have in $(n-1)^{\text {th }}$ layer above identity in subgroups lattice diagram are

$$
\begin{gathered}
H_{1}=Z_{p_{1}} \times\{0\} \times\{0\} \times\{0\} \times\{0\}_{n} \\
H_{2}=\{0\} \times Z_{p_{2}} \times\{0\} \times\{0\} \times\{0\}_{n} \\
\vdots \\
H_{i}=\{0\} \times\{0\} \times Z_{p_{i}} \times\{0\} \times\{0\}_{n} \\
\vdots \\
H_{n}=\{0\} \times\{0\} \times\{0\} \times\{0\} \times Z_{p_{n}}
\end{gathered}
$$

Thus by inclusion exclusion principal, we have $\left(\begin{array}{c}n \\ n-1\end{array}\right)=n$. Similarly for $(n-2)^{n d}$ layer, we have 


$$
\begin{gathered}
H_{12}=Z_{p_{1}} \times Z p_{2} \times\{0\} \times \cdots \times\{0\}_{n}, \\
H_{13}=Z_{p_{1}} \times\{0\} \times Z_{p_{3}} \times\{0\} \times \cdots \times 0_{n}, \\
\vdots \\
H_{1 n}=Z_{p_{1}} \times\{0\} \times \cdots \times\{0\} \times Z_{p_{n}}, \\
H_{23}=\{0\} \times Z_{p_{2}} \times Z_{p_{3}} \times\{0\} \times \cdots \times 0_{n}, \\
H_{24}=\{0\} \times Z_{p_{2}} \times\{0\} \times Z_{p_{4}} \times\{0\} \times \cdots \times\{0\}_{n}, \\
\vdots \\
H_{(n-1) n}=\{0\} \times\{0\} \times \cdots \times\{0\} \times Z_{p_{n-1}} \times Z_{p_{n}}
\end{gathered}
$$

So, it is easy to see that number of subgroups of $G$ on $(n-2)^{n d}$ layer is

$$
\left(\begin{array}{c}
n \\
n-2
\end{array}\right)=\frac{n !}{2 !(n-2) !}
$$

The subgroups of $G$ in $r^{t h}$ layer on the lattice subgroup by the inclusion exclusion principle are given by,

$$
\left(\begin{array}{l}
n \\
r
\end{array}\right)=\frac{n !}{r !(n-r) !} .
$$

Similarly, in $1^{\text {st }}$ layer below group $G$, we have $\left(\begin{array}{c}n \\ n-1\end{array}\right)=n$.

Corollary 3.8 Let $H$ be a subgroup of $G=Z_{p_{1}} \times Z_{p_{2}} \times \cdots \times Z_{p_{n}}$ where $p_{1}, p_{2}, \ldots, p_{n}$ are $n$ distinct primes, in $k^{\text {th }}$ layer $(1 \leq k \leq n)$, below $G$ in the lattice diagram. Then the number of subgroups containing a particular subgroup $H$, in $r^{\text {th }}$ layer are ${ }^{k} C_{r}$, where $1 \leq r \leq k$. Furthermore, the total number of subgroups of $G$ containing $H$ are $\sum_{i=1}^{k} C_{i}$.

Proof: Let $H_{n}$ be the identity subgroup of $G$ present in $n^{\text {th }}$ layer i.e., $H=$ $\{e\}=\{0\} \times\{0\} \times \ldots \times\{0\}$ upto $n$ times, then by above theorem , the number of subgroups containing $H_{n}$ are ${ }^{n} C_{r}, \forall r=1,2, \ldots, n-1$. \& applying the theorem 3.7 ,follows the result. Now, for $H_{n-1}$ be a subgroup of $G$ present in $(n-1) t h$ layer, then $H_{n-1}$ must be of the form

$$
H_{n-1}=\left\{\prod_{i=1}^{n} Z_{p_{i}} \mid Z_{p_{i}}=0 \text { for }(n-1) \text { positions }\right\}
$$


Now $H_{n-1}$ is contained in all those subgroups in $r^{\text {th }}$ layer $(1 \leq r \leq n-2)$ which has same positions for non-zero $Z_{p_{i}}$ as in $H_{n-1}$. Then the remaining zeroes of $H_{n}$ can be arranged in ${ }^{n-1} C_{r}$ ways for each layer above and each of these new arranged subgroups of $r^{\text {th }}$ layer contain $H_{n-1}$. Thus summing all these ${ }^{n-1} C_{r}$ subgroups in each $r^{\text {th }}$ layer, we obtain total number of subgroups containing $H_{n-1}$. Similarly for the subgroup $H_{k}$ present in $k^{t h}$ layer of the form

$$
H_{k}=\left\{\prod_{i=1}^{n} Z_{p_{i}} \mid Z_{p_{i}}=0 \text { for } k \text { positions }\right\}
$$

is contained in those subgroups of $r^{\text {th }}$ layer which has the same positions for non-zero $Z_{p_{i}}$ as in $H_{k}$. Therefore, the remaining zeroes can be arranged in ${ }^{k} C_{r}$ ways for each layer above and $\sum_{r=1}^{k}{ }^{k} C_{r}$ will give total number of subgroups containing $H_{k}$.

Remark 3.9 The subgroup $\mathrm{H}_{2}$ present in $2^{\text {nd }}$ layer below $\mathrm{G}$ of the lattice diagram is of the form

$$
H_{2}=\left\{\prod_{i=1}^{n} Z_{p_{i}} \mid Z_{p_{i}}=0 \text { for } 2 \text { positions }\right\}
$$

and therefore can be arranged $\mathrm{in}^{2} C_{r}$ ways $(1 \leq r<2)$, the result follows. And the subgroup

$$
H_{1}=\left\{\prod_{i=1}^{n} Z_{p_{i}} \mid Z_{p_{i}}=0 \text { for } 1 \text { position }\right\}
$$

is only contained in $G$.

Corollary 3.10 The number of fuzzy subgroups of $G=Z_{p_{1}} \times Z_{p_{2}} \times \cdots \times Z_{p_{n}}$ where $p_{1}, p_{2}, \ldots, p_{n}$ are $n$ distinct primes, is given by

$$
O\left(F_{G}\right)=2 . O\left(F_{p_{1}=\left\{H_{n}\right\}}\right)
$$

where

$$
O\left(F_{p_{1}=H_{n}}\right)=\sum_{r=0}^{n-1}{ }^{n} C_{r}\left\{\sum_{i=0}^{r-1}{ }^{r} C_{i}\left(\sum_{j=0}^{i-1} C_{j}\left(\ldots\left(1+{ }^{2} C_{1}\right)\right)\right)\right\},
$$

where $H_{n}$ is any subgroup present in nth layer and $n>1$.

Proof: We use mathematical induction on n. $O\left(H_{1}\right)=1, O\left(H_{2}\right)=1+{ }^{2} C_{1}=$ $1+2=3$,so the result hold for all $n \leq k$,

$$
O\left(F_{p_{1}=H_{k}}\right)=\sum_{r=0}^{k-1}{ }^{k} C_{r}\left\{\sum_{i=0}^{r-1}{ }^{r} C_{i}\left(\sum_{j=0}^{i-1}{ }^{i} C_{j}\left(\ldots\left(1+{ }^{2} C_{1}\right)\right)\right\}\right.
$$


where $H_{k}$ is subgroup present in $k^{t h}$ layer. We prove for $n=k+1$. The order of $H_{k+1}$ will be sum of orders of all subgroups containing it by $3.4 \& 3.10$,i.e.,

$$
O\left(H_{k+1}\right)=\sum_{r=0}^{k}{ }^{k+1} C_{r} \times O\left(H_{r}\right) .
$$

Where $H_{r}$ is subgroup present in $r^{\text {th }}$ layer $(0 \leq r \leq k)$ Therefore,

$$
O\left(H_{k+1}\right)=\sum_{r=0}^{k}{ }^{k+1} C_{r} \times\left\{\sum_{i=0}^{r-1} C_{i}\left(\sum_{j=0}^{i-1} C_{j}\left(\ldots\left(1+{ }^{2} C_{1}\right)\right)\right\}\right.
$$

Hence the result follows.

Corollary 3.11 Let $G=Z_{p_{1}} \times Z_{p_{2}} \times \cdots \times Z_{p_{n}}$ be an abelian group, then the number of fuzzy subgroups of $G$ are

$$
O\left(F_{G}\right)=2\left[\sum_{i_{1}=0}^{n-1}\left(\begin{array}{l}
n \\
i_{1}
\end{array}\right)\left\{\sum_{i_{2}=0}^{i_{1}-1}\left(\begin{array}{l}
i_{1} \\
i_{2}
\end{array}\right)\left(\sum_{i_{3}=0}^{i_{2}-1}\left(\begin{array}{l}
i_{2} \\
i_{3}
\end{array}\right)\left(\ldots\left(\sum_{i_{n}=0}^{i_{n-1}-1}\left(\begin{array}{c}
i_{n-1} \\
i_{n}
\end{array}\right)\right)\right)\right\}\right],\right.
$$

\section{Fuzzy Subgroups of an Abelian Group of Order $p^{n} q$}

An Abelian Group of order $p^{n} q$, where $p$ and $q$ are distinct primes and $\mathrm{n}$ is any natural number, is a cyclic group of the form $G=Z_{p^{n}} \times Z_{q}$. Using the definition of equivalence as defined above, we determine the number of fuzzy subgroups of $G$.

Theorem 4.1 The number of distinct fuzzy subgroups of $Z p^{n} \times Z q$ are given by

$$
O\left(F_{G}\right)=2 \times O\left(H_{n}\right)
$$

where,

$$
O\left(H_{n}\right)=2^{n-1}(n+2) .
$$

Proof: Since we have two types of subgroup of $G$ of order $p^{l}$ and $p^{l} q$ $(0 \leq l \leq k)$.It is clear from lattice diagram that the subgroup of order $H_{l}$ are shown in first column of lattice diagram of $G$.Therefore, the order of $H_{l}$ is given as $2^{l}$ and $(0 \leq l \leq k)$. Now for the second type of subgroup the order is given by $O\left(H_{l}\right)=2^{l-1}(l+2)$. We prove it by induction on $n$. For $\mathrm{n}=0 \& 1$, we have by $3.10,1=O\left(H_{0}\right)=2^{0-1}(0+2)$ below $G$ and $3=O\left(H_{1}\right)=2^{1-1}(1+2)$ - Assume the statement is true for $n=k$.i.e., $O\left(H_{k}\right)=2^{k-1}(k+2)$. now we shall show that it is true for $n=k+1$.i.e.,

$$
O\left(H_{k+1}\right)=2^{k}(\overline{k+1}+2)
$$


Since we know by theorem 3.4 that order of $H_{k+1}$ will be the sum of orders of all those subgroups which contain $H_{k+1}$. Therefore, adding orders of $H_{k}^{\prime} s$ in $k^{t h}$ layer and sum of all those subgroups in $k-1^{t h}$ to zeroth layer below $G$,we have the order of $H_{k+1}$,

$$
\begin{gathered}
\frac{2^{k}(k+2)}{2}+\frac{2^{k}(k+2)}{2}+2^{k}=\frac{2^{k}(2 k+4)}{2}+2^{k} \\
=2^{k}(k+2)+2^{k}=2^{k}(k+2+1) \\
=2^{k}(\overline{k+1}+2) .
\end{gathered}
$$

Hence the result follows.

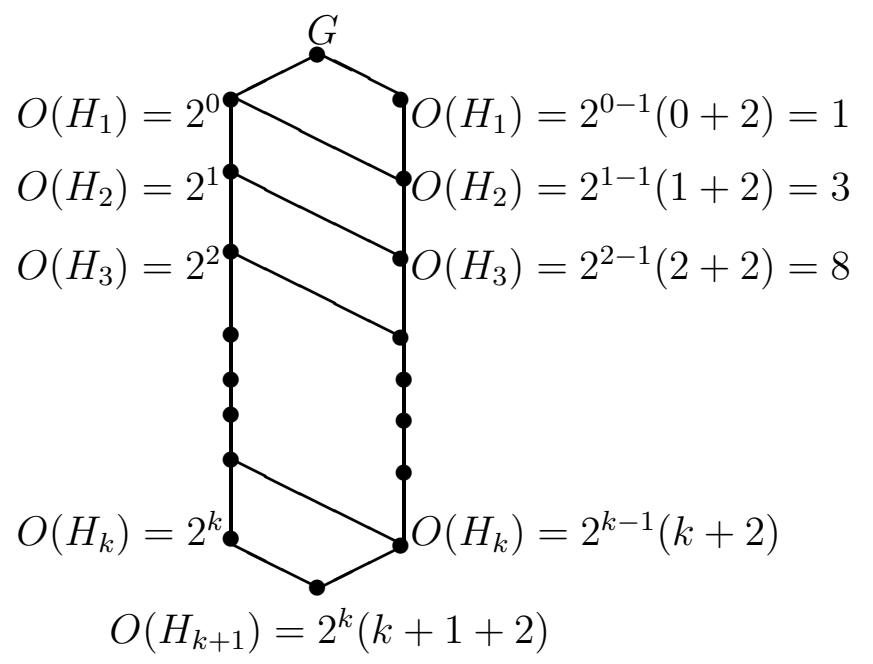

\section{References}

[1] Y.Chen and Y.Jiang.S.Jia, On the number of fuzzy subgroups of finite abelian $p$ - groups, Inter. J. Algebra, 6(5)(2012),233-238.

[2] C.F.Gardiner,A first course in group theory, Springer-Verlag, Berlin,1997.

[3] A.A. Gufar and R. Sulaiman, The number of fuzzy subgroups of finite cyclic groups, Inter. Math. Forum, 06( 20)(2011), 987-994

[4] A.A. Gufar and R. Sulaiman, Counting fuzzy subgroups of symmetric groups $S_{2}, S_{3}$ and alternating group $A_{4}$, J. Quality Measurement and Analysis, 6(1) (2010), 57-63.

[5] I.N.Herstein,Topic in algebra, John Wiley and Sons, New York, 1975.

[6] J.B.Fraleigh, A first course in abstract algebra, Addison-Wesley, London, 1992. 
[7] R.Sulaiman, Constructing fuzzy subgroups of symmetric groups $S_{4}$, Inter. J. Algebra,6(1)(2012),23-28.

[8] V. Murali and B.B.Makamba, Counting the number of fuzzy subgroups of an abelian group of order $p^{n} q^{m}$, Fuzzy Sets Systems, 144 (2004), 459-470.

[9] V.Murali and B.B.Makamba, On an equivalence of fuzzy subgroupsI, Fuzzy Sets and Systems, 123 (2001), 259-264.

[10] V. Murali and B.B. Makamba, On an equivalence of fuzzy subgroups II, Fuzzy Sets and Systems, 136(1) (2003), 93-104.

[11] A.Rosenfeld, Fuzzy groups, J. Math. Anal. and App., 35 (1971), 512-517.

[12] R.Sulaiman, Subgroups lattice of symmetric group $S_{4}$, Inter. J. Algebra, (1) (2012),29-35.

[13] M.Tarnauceanu and L.Bentea, On the number of fuzzy subgroups of finite abelian groups, Fuzzy Sets and Systems, 136 (2003), 93-104.

[14] Y.Zhang and K.Zou, A note on an equivalence relation on fuzzy subgroups, Fuzzy Sets and Systems, 95 (1992),243-247.

\section{Received: October, 2012}

